\title{
Bonding Orthodontic Ceramic Brackets to Ceramic Restorations: Evaluation of Different Surface Conditioning Methods
}

\author{
Andreas Faltermeier*, Claudia Reicheneder, Peter Götzfried, Peter Proff \\ Department of Orthodontics, University Medical Centre of Regensburg, Regensburg, Germany. \\ Email: *Andreas.Faltermeier@klinik.uni-regensburg.de
}

Received May $10^{\text {th }}, 2013$; revised June $12^{\text {th }}, 2013$; accepted June $23^{\text {rd }}, 2013$

Copyright (c) 2013 Andreas Faltermeier et al. This is an open access article distributed under the Creative Commons Attribution License, which permits unrestricted use, distribution, and reproduction in any medium, provided the original work is properly cited.

\begin{abstract}
The purpose of this study was to compare the shear bond strength and Adhesive Remnant Index of four different veneering ceramic materials to ceramic brackets. Additionallly, a further aim of this study was to overcome the etching using hydrofluoric acid which is noxious and could seriously damage the corneas of the eyes. Two surface conditioning methods of four ceramic materials before bonding brackets were examined: in group 1 an air particle abrasion with 25 $\mu \mathrm{m}$ aluminium trioxide ( 4 seconds at a pressure of 2.5 bars) and subsequently a silane coupling agent (Espe Sil, 3M Unitek, Monrovia, USA) was applicated on one side of each ceramic specimen (10 per group). In group 2 one side of each sample (20 per group) was etched with 37.0 per cent orthophosphoric acid for two minutes and was followed by a silane application (Espe Sil, 3M Unitek, Monrovia, USA). After this procedure the self-ligating ceramic brackets Clarity SL (3M Unitek, Monrovia, USA) brackets were bonded to the ceramic blocks and a thermocycling process started $\left(5^{\circ} \mathrm{C}\right.$ $55^{\circ} \mathrm{C}, 6000$ cycles). Then, shear bond strength and Adhesive Remnant Index (ARI) were measured. To determine statistical differences Oneway-ANOVA and Tukey Post-hoc test were performed. Hydrofluoric acid seems not to be justifiable anymore for preparing the surface of dental ceramic restorations before bracket bonding. Sandblasting with 25 $\mu \mathrm{m}$ aluminium trioxide and the use of orthophosphoric acid (37.0 per cent) seem to prepare the surface of ceramic restoration sufficiently before ceramic bracket bonding. The found level of shear bond strength values seems to be sufficient for bonding ceramic brackets to ceramic restorations.
\end{abstract}

Keywords: Ceramic Restorations; Ceramic Brackets; Aluminium Trioxide; Orthophosphoric Acid

\section{Introduction}

Dental ceramics are nowadays often used as restorative material as veneers, crowns and bridges because of their aesthetic appearance, their outstanding mechanical properties and their biocompatibility. The increased demand by adults for orthodontic treatment results in the necessity for orthodontics to attach brackets and tubes on ceramic restorations. However, the difficulty of orthodontic bracket bonding is its semipermanent nature. Bond strength should be high enough to resist accidental debonding during treatment but also low enough to remove the bracket from the ceramic without generating excessive force which might damage the peridentium of the tooth or the restoration [1,2]. As a result of the basic investigations of Buonocore (1955) the direct bonding te-

*Corresponding author. chnique has revolutionized bonding brackets to teeth [3]. Over the years a lot of attention has been paid to improving the acid-etching technique, primers and adhesives.

Ceramic restorations, such as ceramic crowns or veneers, which are adhesively bonded to teeth, can be nowadays more often found in orthodontic patients because of an increasing number of adult patients who ask for a treatment [2]. Several methods have been suggested to strengthen the bond strength between ceramics and brackets: it was described to roughen the porcelain with diamond burs, green stones or abrasive disks [2,4]. Others described for getting more retention to cut a retention cavity in the ceramic surface [5]. Nevertheless, all these procedures damage the glazed surface of restoration. Another method of roughening the ceramic surface is to apply acids or sandblasting [6]. The use of strong acids to roughen the ceramic surface was suggested by Calamia 
[7]. As acids hydrofluoric acid and orthophosphoric acid have been suggested. 9.6 per cent hydrofluoric acid is able to create a series of surface pits by preferential dissolution of the glass phase from the ceramic matrix [6]. Nevertheless, it is described as an acid which is extremely dangerous because of its corrosive character and the danger of causing severe trauma to soft tissues and tooth substance $[2,8]$.

Therefore, the question arises which procedure should be applied for bonding brackets to ceramic restorations. It has been suggested by several authors [5,6,9] to utilize hydrofluoric acid for preparing the ceramic surface. Hydrofluoric acid is best known for its ability to dissolve glass by reacting with $\mathrm{SiO}_{2}$, the major component of most glass, to form silicon tetrafluoride gas and hexafluorosilicic acid. This property has been known since the 17th century, even before hydrofluoric acid had been prepared in large quantities by Carl Wilhelm Scheele in 1771 [10]. It is also unique in its ability to dissolve many metal and semimetal oxides [5]. The danger in handling hydrofluoric acid is extreme, as skin saturation with the acid in areas of only 25 square inches $\left(160 \mathrm{~cm}^{2}\right)$ may be relatively painless, yet ultimately fatal. High concentrations of hydrofluoric acid and hydrogen fluoride gas will also quickly destroy the corneas of the eyes [2,4].

Consequently, the purpose of this study was to compare the shear bond strength and Adhesive Remnant Index of four different veneering ceramic materials to ceramic brackets (Figure 1). Additionallly, a further aim of this study was to overcome the etching using hydrofluoric acid which is noxious and could hardly damage the corneas of the eyes. Instead of that air particle abrasion with $25 \mu \mathrm{m}$ aluminium trioxide, silane coupling application and etching with 37.0 per cent orthophosphoric acid as pre-treatment procedures of the veneering ceramics before bonding was investigated.

\section{Material and Methods}

Two surface conditioning methods of the ceramic blocks

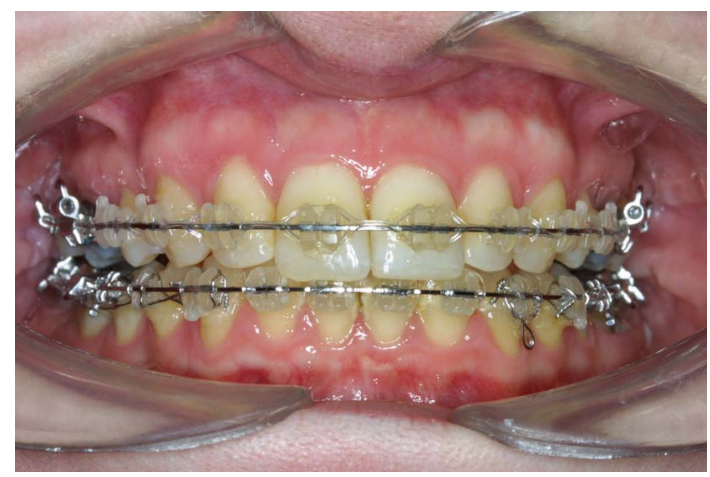

Figure 1. Ceramic brackets bonded to ceramic restorations in-vivo. before bonding brackets were investigated: in group 1 an air particle abrasion with $25 \mu \mathrm{m}$ aluminium trioxide (4 seconds at a pressure of 2.5 bars) and subsequently a silane coupling agent (Espe Sil, 3M Unitek, Monrovia, USA) was applicated on one side of each ceramic specimen (20 per group). In group 2 one side of each sample (20 per group) was etched with 37.0 per cent orthophosphoric acid for two minutes and was followed by a silane application (Espe Sil, 3M Unitek, Monrovia, USA).

A total of 200 ceramic blocks with the dimensions of $10 \times 10 \times 10 \mathrm{~mm}$ were manufactured. The following sintered ceramics were investigated: Vita Omega 900 (VITA Zahnfabrik, Bad Säckingen, Germany), Symbio Ceram (DeguDent, Haunau, Germany), Imagine Reflex (Wieland, Pforzheim, Germany), Hera Ceram Sun (Heraeus Kulzer; Hanau, Germany) and Ducera Gold (DeguDent, Haunau, Germany).

The exact temperatures of each sintered ceramics during the manufacturing process are shown in Table 1. The ceramic blocks were cleaned and polished with burnishers to attain a high gloss.

After this procedure the brackets were bonded to the ceramic blocks as follows: the conventional primer Transbond XT Primer (3M Unitek, Monrovia, USA) was applied, gently thinned with air and light-cured for 20 seconds (Ortholux LED, 3M Unitek). After this procedure the self-ligating ceramic brackets Clarity SL (3M Unitek, Monrovia, USA) were bonded to the ceramic specimens using the adhesive Transbond XT (3M Unitek, Monrovia, USA). All brackets were placed centrally on the prepared surfaces of the samples. The excess resin was carefully removed from the tooth using a dental probe. The samples were then light-cured with a light emitting diode (LED) curing device (Ortholux LED, 3M Unitek) for 20 seconds. All brackets were bonded by the same operator.

To simulate the moisture and temperature changes in the oral environment all samples were exposed to thermocycling 24 hours after preparation (Figure 2). All groups were alternatively flooded every $2 \mathrm{~min}$. with warm $\left(55^{\circ} \mathrm{C}\right)$ and cold $\left(5^{\circ} \mathrm{C}\right)$ distilled water for $6000 \mathrm{cy}$ cles in a mastication device [11].

Measurement of Shear Bond Strength: Specimens were stressed to failure, shear strength, in a universal testing machine (Instron ${ }^{\circledR}$ 5542; Instron Structural Testing Systems GmbH; Pfungstadt; Germany) at a crosshead speed of $1 \mathrm{~mm}$ per min. The critical maximum stress prior to failure was determined for statistical analysis. The SBS was evaluated using the formula: $\sigma$ shear $=$ Fmax/A bracket base surface. The clear impression of the bracket base design guaranteed in most cases a high level of good quality results. The surface area of the bracket bases was determined by measuring length and width and computing the mean area [2]. 
Table 1. Manufacturing parameters of the sintered ceramic groups.

\begin{tabular}{cccc}
\hline Ceramic brand & Preheating temperature $\left[{ }^{\circ} \mathrm{C}\right]$ & Burning temperature $\left[{ }^{\circ} \mathrm{C}\right]$ & Holding time $[\mathrm{s}]$ \\
\hline Vita Omega 900 & 600 & 930 & 60 \\
Symbio Ceram & 575 & 820 & 60 \\
Imagine Reflex & 575 & 900 & 120 \\
Hera Ceram Sun & 600 & 860 & 60 \\
Ducera Gold & 450 & 785 & 60 \\
\hline
\end{tabular}

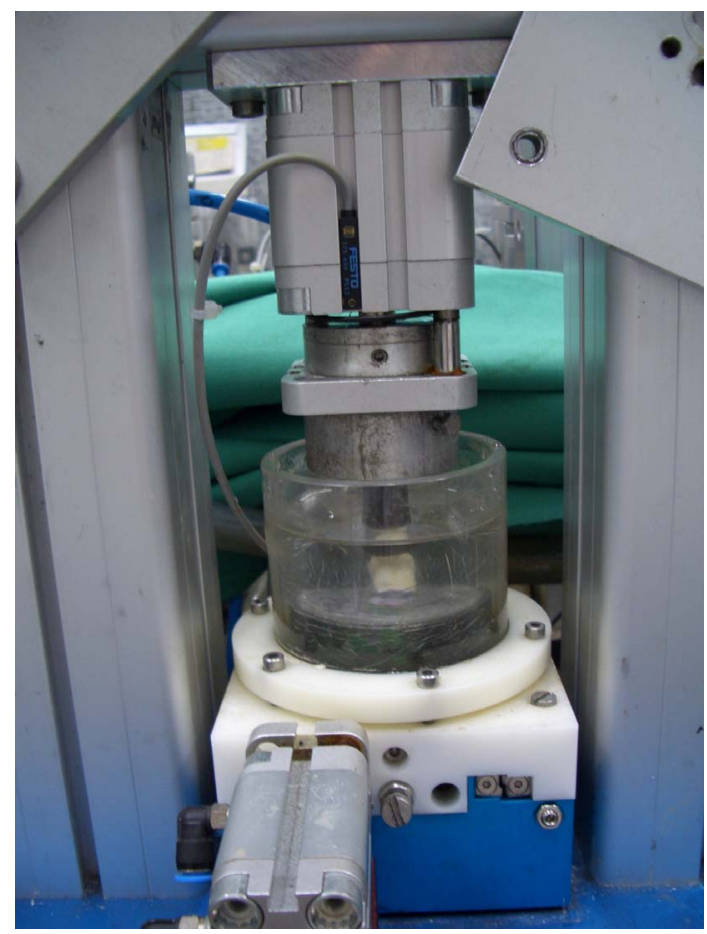

Figure 2. Simulation of the moisture and temperature changes in the oral environment in a mastication device with warm $\left(55^{\circ} \mathrm{C}\right)$ and cold $\left(5^{\circ} \mathrm{C}\right)$ distilled water $(6000$ cycles).

Additionally, the Adhesive Remnant Index (ARI) was determined. The classification of the ARI-index was as follows: "0" means that there is no composite left on the enamel. The score of " 1 " shows, that it remains less than $50 \%$ of the composite on the enamel. The score of "2" indicates that more than $50 \%$ of the composite remained on the enamel. And the score of " 3 " means that all composite remained on the enamel.

To determine statistical differences Kruskal-Wallis test and Mann-Whitney U-test were performed. Means and standard deviations were calculated. The level of significance was set at $\alpha=0.05$.

\section{Results and Discussion}

In Table 2 ARI scores of the different ceramic groups are listed. Results of shear bond strength measurements after etching with 37.0 per cent orthophosphoric acid are
Table 2. ARI (Adhesive Remnant Index) of different ceramic groups.

\begin{tabular}{ccccc}
\hline ARI score & 0 & 1 & 2 & 3 \\
\hline Vita Omega 900 sandblasted $^{*}$ & 13 & 0 & 4 & 3 \\
Vita Omega 900 etched $^{*}$ & 14 & 1 & 3 & 2 \\
Symbio Ceram sandblasted $^{*}$ & 2 & 6 & 2 & 10 \\
Symbio Ceram etched $^{*}$ & 1 & 4 & 3 & 12 \\
Imagine Reflex sandblasted $_{\text {Imagine Reflex etched }}^{*}$ & 7 & 3 & 4 & 6 \\
Hera Ceram Sun sandblasted $^{*}$ & 8 & 2 & 2 & 8 \\
Hera Ceram Sun etched $^{*}$ & 6 & 1 & 2 & 13 \\
Ducera Gold sandblasted $^{*}$ & 10 & 4 & 3 & 3 \\
Ducera Gold etched $^{*}$ & 9 & 2 & 4 & 5 \\
\hline
\end{tabular}

etched*: etched with 37.0 per cent orthophosphoric acid for 1 minute. ARI scores: "0": no composite remained on the enamel; " 1 ": less than $50 \%$ of the composite remained on the enamel; " 2 ": more than $50 \%$ of the composite remained on the enamel; " 3 ": all composite remained on the enamel.

depicted in Figure 3. In Figure 4 the results of shear bond strength measurements after air particle abrasion with $25 \mu \mathrm{m}$ aluminium trioxide are demonstrated. The statistical analysis of the data obtained in our study reveals that there is no significant enhancement of shear bond strength using sandblasting with $25 \mu \mathrm{m}$ aluminium trioxide in comparison to using orthophosphoric acid (37.0 per cent) as surface conditioning method of ceramic restorations ( $p>0.05$, Table 3$)$. Almost the same result could be observed when ARI scores are compared: No statistical different between the two surface conditioning methods could be measured ( $\mathrm{p}>0.05$, Table 3). Median values of shear bond strength are shown in Table 4. The highest shear bond values could be observed using the ceramic Imagine Reflex after air particle abrasion with $25 \mu \mathrm{m}$ aluminium trioxide as surface conditioning method (230.5 N, Table 4).

The ceramic restoration Ducera Gold revealed the lowest shear bond values of all groups after sandblasting with aluminium trioxide (127.8 N, Table 4).

The treatment of adult patients is becoming more and more vital in orthodontics at the present time. The operator is often faced with the problem of ceramic restorations, especially in the anterior region, of elderly adult 


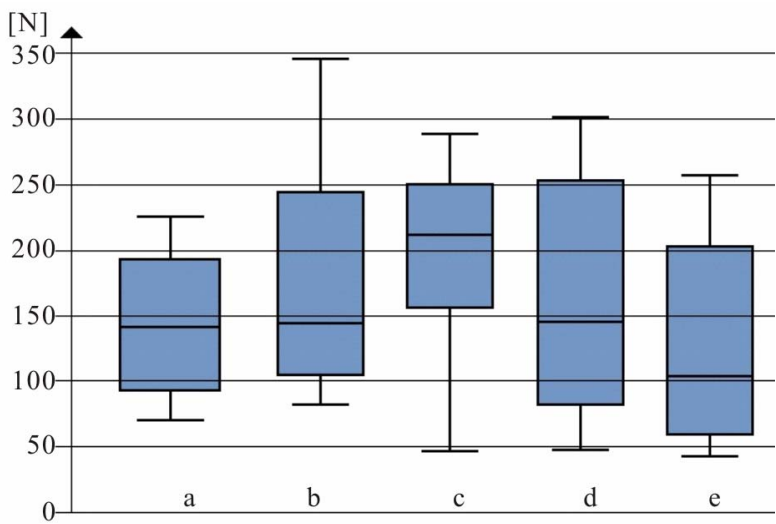

Figure 3. Shear bond strength $[\mathrm{N}]$ of different ceramic groups after etched with 37.0 per cent orthophosphoric acid before bracket bonding: a: Vita omega 900; b: Symbio ceram; c: Imagine reflex; d: Hera ceram sun; e: Ducera gold.

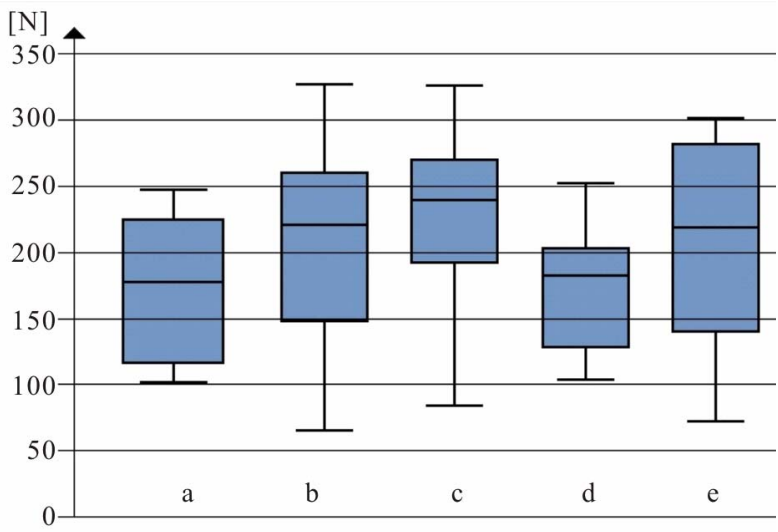

Figure 4. Shear bond strength $[\mathrm{N}]$ of different ceramic groups after air particle abrasion with $25 \mu \mathrm{m}$ aluminium trioxide before bracket bonding: a: Vita omega 900; b: Symbio ceram; c: Imagine reflex; d: Hera ceram sun; e: Ducera gold.

patients. Most of the veneering ceramic materials available on the market are feldspatic with dispersed crystalline phase in the glass matrix. The structure of veneering ceramic has been described as an amorphous and glassy matrix that consists of a random network of cross-linked silica in a tetrahedral arrangement, which is embedded in varying amounts of undissolved feldspatic and leucite crystals [2,12]. Nevertheless, hydrothermal ceramics, so called glass ceramics, with innovative chemical and physical properties become more and more important. In this material the quartz lattice, which forms the vitreous phase, is modified by adding alkali oxides, which lower the melting temperature, increases the coefficient of expansion [2,10]. For ceramic surface treatment, the acid can react with the glassy matrix, which is selectively removed and the crystalline structure is exposed. This leads to rougher surface of the veneering ceramic which
Table 3. Statistical analysis.

\begin{tabular}{clc}
\hline & SBS & ARI index \\
\hline Vita Omega 900 sandblasted vs. etched $^{*}$ & n.s. & n.s. \\
Symbio Ceram sandblasted vs. etched & n.s. & n.s. \\
Imagine Reflex sandblasted vs. etched & n.s. & n.s. \\
Hera Ceram Sun sandblasted vs. etched ${ }^{*}$ & n.s. & n.s. \\
Ducera Gold sandblasted vs. etched $^{*}$ & n.s. & n.s. \\
\hline
\end{tabular}

Statistical analysis (Kruskal-Wallis test, Mann-Whitney U-test) of shear bond strength (SBS) and Adhesive Remnant Index (ARI). n.s.: not significant. Level of significance: $p=0.05$. etched ${ }^{*}$ : etched with 37.0 per cent orthophosphoric acid for 1 minute, no significant difference ( $p>0.05$ ) between sandblasted and etched ceramics within a material group and between the groups were measured.

Table 4. Median-values of different ceramic groups. Shear bond strength: SBS.

\begin{tabular}{|c|c|}
\hline Ceramic groups: & SBS [MPa] \\
\hline Vita Omega 900 sandblasted & 163.4 \\
\hline Vita Omega 900 etched $^{*}$ & 143.8 \\
\hline Symbio Ceram sandblasted & 209.3 \\
\hline Symbio Ceram etched ${ }^{*}$ & 175.1 \\
\hline Imagine Reflex sandblasted & 230.5 \\
\hline Imagine Reflex etched ${ }^{*}$ & 195.9 \\
\hline Hera Ceram Sun sandblasted & 174.1 \\
\hline Hera Ceram Sun etched* & 161.4 \\
\hline Ducera Gold sandblasted & 127.8 \\
\hline Ducera Gold etched ${ }^{*}$ & 143.7 \\
\hline
\end{tabular}

means more micromechanical retention [10].

For that reason we decided to use in this study orthophosphoric acid instead of hydrofluoric acid with the aim to demonstrate that hydrofluoric acid is not necessary for bonding brackets to ceramic restoration. It was demonstrated that orthophosphoric acid with a concentration of 37.0 per cent is not able to etch a ceramic surface and, consequently, does not produce physical or topographical changes on porcelain. On the other hand, orthophosphoric acid has the ability to neutralize the alkalinity of the absorbed water layer, which is present on all ceramic restorations in the mouth and, thereby, improve the chemical activity of any silane primer when subsequently applied $[2,8]$. Silane molecules, after being hydrolized to silanol, are able to form a polysiloxane network or hydroxyl groups to cover the silica surface. Monomeric ends of silane molecules react with the methacrylate groups of the adhesive resins by free radical polymerization [13,14].

The aim of this study was to compare the shear bond strength and Adhesive Remnant Index of four different veneering ceramic materials to ceramic brackets and to examine whether it is possible to overcome the etching 
using hydrofluoric acid which is noxious. Instead of this treatment, air particle abrasion with $25 \mu \mathrm{m}$ aluminium trioxide, silane coupling application and etching with 37.0 per cent orthophosphoric acid as pre-treatment procedures of the veneering ceramics before bonding was investigated [2].

On the basis of the results of this study, the method of sandblasting with $25 \mu \mathrm{m}$ aluminium trioxide the surface of the ceramic restoration could be rejected because the method of using only orthophosphoric acid (37.0 per cent) revealed comparable results and is much easier to apply. The ARI scores measured in this study confirm this conclusion. Additionally, the use of hydrofluoric acid seems not to be justifiable anymore for preparing the surface of dental ceramic restorations before bracket bonding. The danger in handling hydrofluoric acid is extreme, because skin and corneas of the eyes could be severely damaged by contact. The results of the present study show that median values of 127.8 - 230.5 MPa could be reached using only orthophosphoric acid (37.0 per cent). This level of shear bond strength is described to be sufficient for bracket bonding and to avoid accidental bracket debonding $[2,5,6,9]$. The results of the present study show that there is no significant enhancement of shear bond strength and ARI scores using sandblasting with 25 $\mu \mathrm{m}$ aluminium in comparison to using orthophosphoric acid (37.0 per cent) as surface conditioning method of ceramic restorations.

\section{Conclusion}

Hydrofluoric acid should not be used anymore for preparing the surface of dental ceramic restorations before bracket bonding. Sandblasting with $25 \mu \mathrm{m}$ aluminium trioxide and the use of orthophosphoric acid (37.0 per cent) prepare the surface of ceramic restoration sufficiently before ceramic bracket bonding. The found level of shear bond strength values is sufficient for bonding ceramic brackets to ceramic restorations.

\section{REFERENCES}

[1] M. G. Buonocore, "Retrospectives on Bonding," Dental Clinics of North America, Vol. 25, No. 2, 1981, pp. 241-255.

[2] A. Faltermeier, P. Roemer, C. Reicheneder, P. Proff and T. Klinke, "The Influence of Ceramic Restorations before metal Bracket Bonding,” Materials Sciences and Applications, Vol. 3, No. 1, 2012, pp. 1-5.

[3] M. G. Buonocore, "A Simple Method of Increasing the Adhesion of Acrylic Filling Materials to Enamel Surfaces,” Journal of Dental Research, Vol. 34, No. 6, 1955, pp. 849-853. doi:10.1177/00220345550340060801

[4] P. Schmage, I. Nergiz, W. Herrmann and M. Özcan, "Infulence of Various Surface-Conditioning Methods on the Bond Strength of Metal Brackets to Ceramic Surfaces," American Journal of Orthodontics and Dentofacial Orthopedics, Vol. 123, No. 5, 2003, pp. 540-546. doi:10.1016/S0889-5406(02)56911-0

[5] S. Karan, T. Büyükyilmaz and M. S. Toroglu, "Orthodontic Bonding to Several Ceramic Surfaces: Are There Acceptable Alternatives to Conventional Methods,” American Journal of Orthodontics and Dentofacial Orthopedics, Vol. 132, No. 2, 2007, pp. 144.e7-144.e14.

[6] G. Trakyali, Ö. Malkondu, E. Kazazoglu and T. Arun, "Effects of Different Silanes and Acid Concentrations on Bond Strength to Porcelain Surfaces," European Journal of Orthodontics, Vol. 31, No. 4, 2009, pp. 402-406. doi:10.1093/ejo/cjn118

[7] J. R. Calamia, "Etched Porcelain Facial Veneers: A New Treatment Modality Based on Scientific and Clinical Evidence," New York Journal of Dentistry, Vol. 53, No. 6, 1983, pp. 225-230.

[8] T. Hayakawa, K. Horie, M. Aida, H. Kanaya, T. Kobayashi and Y. Murata, "The Influence of Surface Conditions and Silane Agents on the Bond of Resin to Dental Porcelain,” Dental Materials, Vol. 8, No. 4, 1992, pp. 238-240. doi:10.1016/0109-5641(92)90092-Q

[9] M. Özcan, P. K. Vallittu, T. Peltomäki, M. C. Huysmans and W. Kalk, "Bonding Polycarbonate Brackets to Ceramic: Effects of Substrate Treatment on Bond Strength," American Journal of Orthodontics and Dentofacial Orthopedics, Vol. 126, No. 2, 2004, pp. 220-227. doi:10.1016/j.ajodo.2003.06.015

[10] T. Türk, D. Sarac, Y. Sarac and S. Elekdag-Türk, "Effects of Surface Conditioning on Bond Strength of Metal Brackets to All-Ceramic Surfaces,” European Journal of Orthodontics, Vol. 28, No. 5, 2006, pp. 450-456. doi:10.1093/ejo/cj1010

[11] M. Rosentritt, T. Plein, C Kolbeck, M. Behr and G. Handel, "In Vitro Force and Marginal Adaption of Ceramic Crowns Fixed on Natural Artificial Teeth,” International Journal of Prosthodontics, Vol. 13, No. 5, 2000, pp. 387391.

[12] B. M. Bourke and W. P. Rock, "Factors Affecting the Shear Bond Strength of Orthodontic Brackets to Porcelain,” British Journal of Orthodontics, Vol. 26, No. 4 1999, pp. 285-290. doi:10.1093/ortho/26.4.285

[13] J. Daub, D. W. Berzins, B. J. Linn and T. G. Bradley, "Bond Strength of Direct and Indirect Bonded Brackets after Thermocycling," Angle Orthodontist, Vol. 76, No. 2, 2006, pp. 295-300.

[14] I. Gillis and M. Redlich, "The Effect of Different Porcelain Conditioning Techniques on Shear Bond Strength of Stainless Steel Brackets," American Journal of Orthodontics and Dentofacial Orthopedics, Vol. 114, No. 4, 1998, pp. 387-392. doi:10.1016/S0889-5406(98)70183-0 